\title{
A theranostic nanocomposite with integrated black phosphorus nanosheet, Fe304@MnO2-doped upconversion nanoparticles and chlorin for simultaneous multimodal imaging, highly efficient photodynamic and photothermal therapy
}

Zhang, Qicheng; Wang, Wentao; Zhang, Ming; Wu, Fan; Zheng, Tao; Sheng, Bulei; Liu, Yihan; Shen, Jian; Zhou, Ninglin; Sun, Yi

Published in:

Chemical Engineering Journal

Link to article, DOI:

10.1016/j.cej.2019.123525

Publication date:

2020

Document Version

Peer reviewed version

Link back to DTU Orbit

Citation (APA):

Zhang, Q., Wang, W., Zhang, M., Wu, F., Zheng, T., Sheng, B., Liu, Y., Shen, J., Zhou, N., \& Sun, Y. (2020). A theranostic nanocomposite with integrated black phosphorus nanosheet, $\mathrm{Fe} \mathrm{O}$ @MnO -doped upconversion nanoparticles and chlorin for simultaneous multimodal imaging, highly efficient photodyramic and photothermal therapy. Chemical Engineering Journal, 391, [123525]. https://doi.org/10.1016/j.cej.2019.123525

\section{General rights}

Copyright and moral rights for the publications made accessible in the public portal are retained by the authors and/or other copyright owners and it is a condition of accessing publications that users recognise and abide by the legal requirements associated with these rights.

- Users may download and print one copy of any publication from the public portal for the purpose of private study or research.

- You may not further distribute the material or use it for any profit-making activity or commercial gain

- You may freely distribute the URL identifying the publication in the public portal 


\section{A Theranostic Nanocomposite with Integrated Black Phosphorus Nanosheet, $\mathrm{Fe}_{3} \mathrm{O}_{4} @ \mathrm{MnO}_{2}$-doped Upconversion Nanoparticles and Chlorin for Simultaneous}

Multimodal Imaging, Highly Efficient Photodynamic and Photothermal Therapy

Qicheng Zhang ${ }^{a+}$, Wentao Wang ${ }^{\mathrm{a}, \mathrm{b}+}$, Ming Zhang,b*, Fan Wu ${ }^{\mathrm{a}}$, Tao Zheng ${ }^{\mathrm{b}}$, Bulei Sheng ${ }^{\mathrm{c}}$, Yihan Liu $^{\mathrm{a}}$, Jian Shen ${ }^{\mathrm{a}}$, Ninglin Zhou ${ }^{\mathrm{a} *}$, Yi Sun ${ }^{\mathrm{b} *}$

${ }^{a}$ Jiangsu Collaborative Innovation Center for Biomedical Functional Materials, School of Chemistry and Materials Science, Nanjing Normal University, Nanjing 210023, P. R. China.

${ }^{b}$ Department of Health Technology, Technical University of Denmark, Kongens Lyngby DK-2800, Denmark.

${ }^{c}$ Department of Food Science, Aarhus University, 8830 Tjele, Denmark

Correspondingauthor: Ming Zhang,mzhan@dtu.dk; NinglinZhou, zhouninglin@njnu.edu.cn; Yi Sun, suyi@dtu.dk

${ }^{+}$These authors contribute equality to this work.

\section{Abstract}

As a novel photosensitizer for photodynamic therapy (PDT), black phosphorus (BP) has attracted increasing attention recently. However, some of its inherent drawbacks hinder its further biomedical applications. As with most conventional photosensitizers, the PDT efficiency of BP is severely limited by the hypoxia tumor microenvironment and low tissue penetration of ultraviolet/visible light. Moreover, BP also faces additional problems, such as mismatch wavelength between photothermal therapy (PTT) and PDT, as well as no imaging ability for cancer diagnosis. Herein, we addressed these challenges through the combination of $\mathrm{Fe}_{3} \mathrm{O}_{4} @ \mathrm{MnO}_{2}$-doped upconversion nanoparticles (UCNPs) and black phosphorus nanosheets (BPNs). By applying polyacrylic acid (PAA) as a chemical crosslinker, polylysine (PL) modified black phosphorus nanosheets were integrated with magnetic $\mathrm{Fe}_{3} \mathrm{O}_{4} @ \mathrm{MnO}_{2}-$ doped $\mathrm{NaYF}_{4}: \mathrm{Yb} / \mathrm{Er} / \mathrm{Nd}$ upconversion nanoparticles (MUCNPs) which could convert near-infrared (NIR) light into visible light. Then, a biocompatible photosensitizer, chlorin e6 (Ce6) was attached 
onto the obtained MUCNPs@BPNs to prepare MUCNPs@BPNs-Ce6 nanocomposites. BPNs and Ce6 were exploited to activate PTT and PDT under a single irradiation light of $808 \mathrm{~nm}$ with strong tissue penetration ability. Meanwhile, the doped $\mathrm{Fe}_{3} \mathrm{O}_{4}$ and $\mathrm{MnO}_{2}$ nanoparticles could provide $\mathrm{T} 1$ and $\mathrm{T} 2$ magnetic resonance (MR) imaging, which could form a multimodal imaging system with fluorescence imaging of $\mathrm{Ce} 6$. In addition, $\mathrm{MnO}_{2}$ could decompose overexpressed $\mathrm{H}_{2} \mathrm{O}_{2}$ in tumor microenvironment to generate $\mathrm{O}_{2}$ to enhance PDT. The magnetic BPNs-based nanocomposites provided multimodal imaging (including MR, fluorescence, optoacoustic, ultrasonic imaging) and displayed dramatically enhanced ability for tumor cell death with visualized synergistic phototherapy, which greatly facilitated the application of BPNs in biomedicine.

Keywords: photodynamic therapy, black phosphorus, photothermal therapy, multimodal imaging, synergistic phototherapy

\section{Introduction}

Photodynamic therapy (PDT) based on the photochemical reaction of a photosensitizer is an emerging clinical strategy for cancer treatment with minimal invasiveness [1-3]. The surface of photosensitizer could generate electrons under suitable light and reduce molecular oxygen into cytotoxic reactive oxygen species (ROS), like singlet oxygen $\left({ }^{1} \mathrm{O}_{2}\right)[4,5]$. By interacting with a variety of biological macromolecules in cells, ROS can cause damage to the cell membrane system and destroy the structure and function of organelles at the target site by oxidative damage, eventually causing apoptosis and necrosis of target cells [6,7].

Recently, black phosphorus (BP) has emerged as a 2D layered photosensitizer [8,9]. As a potential PDT agent, BP possesses ultrahigh surface areas, fascinating photoelectronic properties, high quantum 
yields (up to 0.91) and negligible cytotoxicity [10-13]. The ultra-thin BP nanosheets (BPNs) obtained with the liquid-phase exfoliation by breaking down $\mathrm{BP}$ have shown a strong ability for ${ }^{1} \mathrm{O}_{2}$ production for PDT irradiated at around $660 \mathrm{~nm}$, which could be attributed to their low electron-hole recombination rate and plentiful supply of active sites on the surface [14,15]. Meanwhile, BPNs exhibit high photothermal conversion efficiency irradiated at $808 \mathrm{~nm}$, which means that BPNs can also serve as photothermal therapy (PTT) agents $[16,17]$. Especially, PTT can produce heat in localized tissue to increase blood flow for enhanced $\mathrm{O}_{2}$ transport and, ultimately achieving a synergetic effect with PDT [18]. However, a number of disadvantages of BPNs have hindered their practical application in the biomedical area. For instance, there is a mismatch of wavelengths for making optimal PTT (808 nm) and PDT $(660 \mathrm{~nm})$ work simultaneously. During the actual treatment process, it is difficult and uneconomic to use two light sources for phototherapy, and laser irradiation at $606 \mathrm{~nm}$ also suffers from strong photon intensity attenuation, causing poor tissue penetration depth [19,20]. In addition, like with most conventional photosensitizers, the PDT efficiency of BP is severely limited by the hypoxia tumor microenvironment $[18,21]$. Besides, BPNs usually lack imaging features for cancer diagnosis, which hampers its clinical application in theranostics [22].

One promising solution to overcome these disadvantages is to combine BPNs with multifunctional upconversion nanoparticles (UCNPs). This kind of material can absorb long wavelength photons, which have strong tissue penetration and emit high-energy light with shorter wavelengths to activate photosensitizers [23-25]. Moreover, some mental elements with imaging capabilities $\left(\mathrm{Gd}, \mathrm{Mn}^{2+}\right.$ etc.) were doped into UCNPs for endowing UCNPs with the ability of imaging to build a UCNPs guided multimodal imaging therapeutic systems [26,27]. Lin et al. [28] have successfully conjugated $\mathrm{NaGdF}_{4}: \mathrm{Yb}, \mathrm{Er} @ \mathrm{Yb} @ \mathrm{Nd} @ \mathrm{Yb}$ UCNPs with BPNs for 808 nm laser-mediated 
PDT. However, they did not demonstrate the PTT effect, and the nanosystem had no imaging functionality.

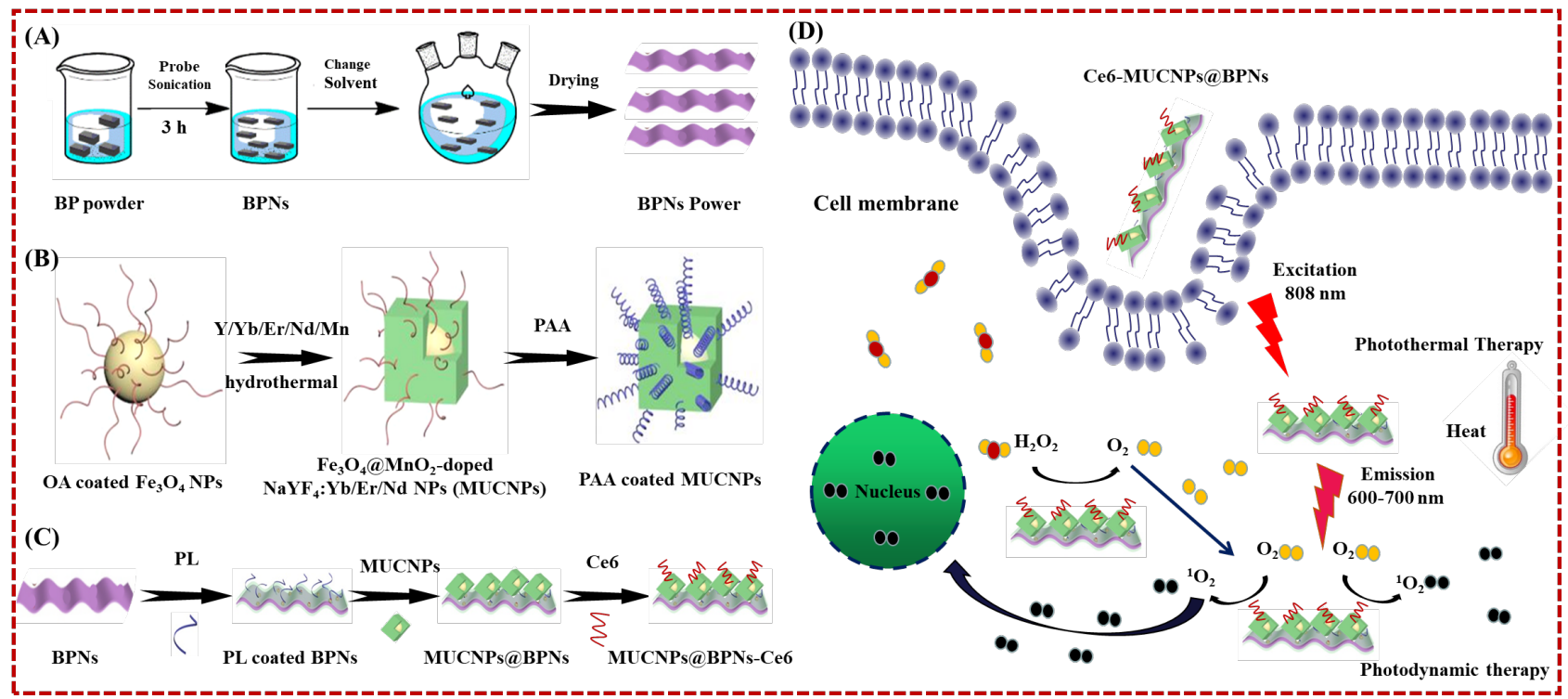

Scheme 1. (A-C) Schematic illustration of the fabrication of BP-based nanoplatforms. (D) Schematic illustration of BP-based nanoplatform for enhanced PDT and PTT therapy upon single $808 \mathrm{~nm}$ laser irradiation.

There are some problems associated with BP serving as PDT agents, such as severe poor $\mathrm{O}_{2}$ production in the tumor environment, low tissue penetration from activating light, mismatch of wavelength between simultaneous PTT and PDT, and lack of imaging functionality in real time. As a solution, we conjugated $\mathrm{Fe}_{3} \mathrm{O}_{4} @ \mathrm{MnO}_{2}$ doped UCNPs with BPNs and fabricated a novel BPNs-based theranostic platform that could simultaneously achieve: 1) PTT/PDT at single 808 laser irradiation; 2) self-support of $\mathrm{O}_{2}$ in tumor microenvironment; 3) multimodal imaging. We innovatively integrated the ultrathin BPNs with the small $\mathrm{Fe}_{3} \mathrm{O}_{4} @ \mathrm{MnO}_{2}$-doped $\mathrm{NaYF}_{4}: \mathrm{Yb} / \mathrm{Er} / \mathrm{Nd}$ UCNPs (MUCNPs) and the chlorin e6 (Ce6) in one nanosystem. As shown in Scheme 1A, the BPNs were firstly obtained by ultrasonic liquid phase stripping from BP powder. The MUCNPs obtained from oleic acid (OA) wrapped $\mathrm{Fe}_{3} \mathrm{O}_{4}$ nanoparticles (NPs) was modified with the strong coordination molecule poly (acrylic acid) (PAA) to acquire good hydrophilicity (Scheme 1B) [29,30]. Then, the polylysine (PL) covered 
BPNs by electrostatic interaction to enhance the stability of BPNs [31] and the functional groups of the two materials were integrated by chemical bond crosslinking. Finally, the Ce6 was loaded onto the nanoplatform (Scheme 1C). Ce6 has high sensitizing efficacy and high fluorescent emission between the wavelengths of 660-670 nm, which can be utilized for both optical imaging and PDT in vivo [3234]. The PTT of BPNs was firstly activated when the nanocomposites were irradiated with $808 \mathrm{~nm}$ laser. Then, the high wavelength laser was adjusted to between $660-670 \mathrm{~nm}$ by the MUCNPs, which was the specific excitation wavelength of PDT for both BPNs and Ce6 [35-37]. The ultraviolet (UV) and visible light emitted from the MUCNPs core upon NIR light irradiation activated the electrons which reacted with the electron acceptors (dissolved $\mathrm{O}_{2}$ from $\mathrm{H}_{2} \mathrm{O}_{2}$ ) to produce ${ }^{1} \mathrm{O}_{2}$. At the same time, the $\mathrm{MnO}_{2}$ transformed from the doped $\mathrm{Mn}^{2+}$ under the high temperature and pressure during the hydrothermal process could efficiently catalyze excessive intracellular $\mathrm{H}_{2} \mathrm{O}_{2}$ in tumor microenvironment to generate $\mathrm{O}_{2}$ [38]. This process was essential to sustain BPNs and Ce6-mediated PDT therapy (Scheme 1D). Additionally, as a result of the reduced $\mathrm{pH}$ of the tumor microenvironment, the obtained $\mathrm{MnO}_{2}$ decomposed to $\mathrm{Mn}^{2+}$ and provided the $\mathrm{T} 1$ and $\mathrm{T} 2$ signal with $\mathrm{Fe}_{3} \mathrm{O}_{4}$ for in vivo magnetic resonance (MR) imaging applications, which we could combine with fluorescence imaging of Ce6 to form a multimodal imaging system. The ROS generation efficiency, photothermal conversion efficiency, tumor therapeutic effect and imaging capabilities were performed well in both in vitro and in vivo experiments and it was found that the prepared MUCNPs@BPNs-Ce6 possessed excellent tumor inhibition efficiency $[39,40]$.

\section{Results and discussion}

The novel theranostic nanoplatform was rationally designed and fabricated by coating MUCNPs 
on amino modified BPNs, and loading Ce6 through coupling the carboxyl groups of the Ce6 molecules and PAA onto MUCNPs with the exposed amino groups on the surface of BPNs. To prepare MUCNPs, OA capped $\mathrm{Fe}_{3} \mathrm{O}_{4}$ with a mean diameter of $15 \mathrm{~nm}$ (Figure 1A) were initially synthesized through a coprecipitation method [41]. Afterwards, the as-synthesized $\mathrm{OA}-\mathrm{Fe}_{3} \mathrm{O}_{4} \mathrm{NPs}$ were performed as seeds, on which the red upconversion luminescent shell $\left(\mathrm{MnO}_{2}\right.$-doped $\left.\mathrm{NaYF}_{4}: \mathrm{Yb} / \mathrm{Er} / \mathrm{Nd}\right)$ was formed by hydrothermal synthesis $[23,42]$. The high-resolution transmission electron microscope (HRTEM) image in Figure 1B shows that the lattice fringes of the $\mathrm{Fe}_{3} \mathrm{O}_{4}$ cores and MUCNPs were $0.26 \mathrm{~nm}$ (inset Figure 1A,B) and $0.31 \mathrm{~nm}$ (Figure 1B) respectively, which ascribed to the (331) lattice planes of the magnetite face-centered cubic structure and the (111) lattice planes of cubic $\mathrm{NaYF}_{4}$. Therefore, this result demonstrated that $\mathrm{MnO}_{2}$-doped $\mathrm{NaYF}_{4}: \mathrm{Yb} / \mathrm{Er} / \mathrm{Nd}$ shells were successfully wrapped onto magnetic seed nanocrystals. The X-ray diffraction (XRD) pattern (Figure S1) further supports the successful preparation of $\mathrm{Fe}_{3} \mathrm{O}_{4}$ (JCPDS Card No.76-1849) and MUCNPs core-shell structures (JCPDS Card No. 16-0342).

The BPNs were prepared by a simple liquid exfoliation technique [43]. The transmission electron microscope (TEM) image shows that the as prepared BPNs were free standing with a 2D nanostructure. It could be observed that the BPNs had a lattice distance of ca. $0.28 \mathrm{~nm}$, and the size ranged from 50 to $100 \mathrm{~nm}$ (Figure 1C). The Raman spectrum from the BP powder and BPNs dispersion showed the three representative BP Raman modes at $362 \mathrm{~cm}^{-1}, 439 \mathrm{~cm}^{-1}$, and $466 \mathrm{~cm}^{-1}$ (Figure S2). After that, PL was grafted on BPNs by electrostatic interaction to provide numerous amino groups for working as reactive functional groups. After PL conjugation, the zeta potential of BPNs increased from -19.5 $\mathrm{mV}$ to $3.8 \mathrm{mV}$ (Figure S3). The positive charge indicated that PL was successfully attached onto BPNs. Afterwards, MUCNPs@BPNs were obtained by assembling BPNs-NH 2 and MUCNPs-PAA onto a theranostic nanoplatform. The morphology and size distribution of the sample were investigated with 
TEM. As shown in Figure 1D, a polymer layer and the MUCNPs were successfully coated onto the BPNs. The quantitative energy dispersive X-ray spectroscopy (EDS) (Figure 1E) confirms that the distribution of the $\mathrm{Fe}, \mathrm{Mn}, \mathrm{Nd}, \mathrm{Yb}$, and $\mathrm{P}$ atoms were homogeneously over the surfaces of the MUCNPs@BPNs. The corresponding EDX spectrum (Figure S4) shows the characteristic energy lines of $\mathrm{Fe}, \mathrm{Mn}, \mathrm{Er}, \mathrm{Yb}, \mathrm{Nd}, \mathrm{Y}, \mathrm{C}, \mathrm{N}$, and $\mathrm{P}$ arising from the MUCNPs and BPNs, and verifies the successful integration of BPNs and UCNPs, which X-ray photoelectron spectroscopy (XPS) also confirmed (Figure 1F). Finally, Ce6 was loaded onto the MUCNPs@BPNs via the formation of BPNsCe6 complexes with covalent interactions. After Ce6 loading, the MUCNPs@BPNs showed red fluorescent and good dispersion in phosphate buffered solution (PBS), Roswell Park Memorial Institute (RPMI) medium, and Dulbecco's modified eagle medium (DMEM) (Figure 1 G). In addition, MUCNPs@BPNs-Ce6 showed high photoluminescence intensity for fluorescence, although the signal was significantly lower compared to free Ce6 at the same concentration (Figure $1 \mathbf{H}$ ).
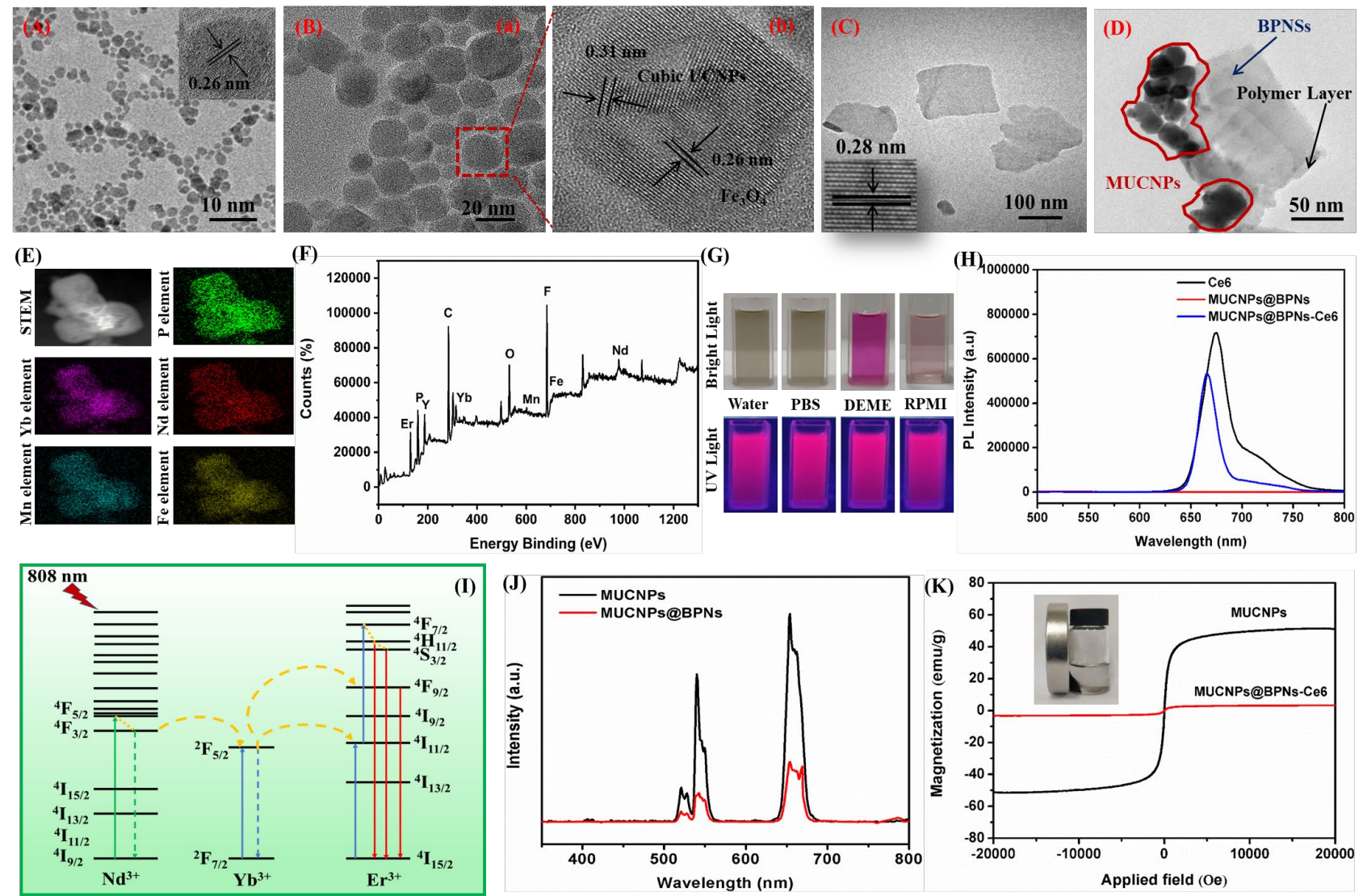
Figure 1. (A) TEM image of $\mathrm{OA}-\mathrm{Fe}_{3} \mathrm{O}_{4}$. (B) TEM (a) and HRTEM (b) image of MUCNPs, (C) TEM image of BPNs. Inset is the HRTEM image of BPNs. (D) TEM image of MUCNPs@BPNs. (E) The corresponding elemental mapping images of MUCNPs@BPNs. (F) XPS spectra of MUCNPs@BPNs. (G) Photograph of MUCNPs@BPNs-Ce6 in water, PBS, DMEM and RPMI under bright or UV light. (H) The photoluminescence spectra of Ce6, MUCNPs@BPNs and MUCNPs@BPNs-Ce6. (I) Upconversion mechanism of MUCNPs@BPNs excited using an 808 nm laser. (J) Upconversion luminescence emission spectra of the MUCNPs and MUCNPs@BPNs under 808 nm irradiation. (K) Magnetic hysteresis loop for MUCNPs and MUCNPs@BPNs-Ce6. Inset is the photographic image of MUCNPs@BPNs-Ce6 solution under a magnet.

In MUCNPs, $\mathrm{Nd}^{3+}$ ions acted as sensitizers to absorb the $808 \mathrm{~nm}$ NIR excitation energy, while $\mathrm{Yb}^{3+}$ ions behaved as bridging ions to receive energy from $\mathrm{Nd}^{3+}$ ions and then transferred it to the $\mathrm{Er}^{3+}$ acceptor ions in a so-called "cascade sensitized UC process" (Figure 1I) [44]. To explore the efficiency of upconversion emission for more efficient PDT, the upconversion luminescence spectra of MUCNPs under $808 \mathrm{~nm}$ NIR light were measured. In Figure 1J, the emissions at 520-560 nm and 645-685 nm were ascribed to ${ }^{4} \mathrm{~S}_{3 / 2} \rightarrow{ }^{4} \mathrm{I}_{15 / 2}$ and ${ }^{4} \mathrm{~F}_{9 / 2} \rightarrow{ }^{4} \mathrm{I}_{15 / 2}$ transitions of $\mathrm{Er}^{3+}[45]$, demonstrating that the efficient energy transferred from UCNPs to BPNs and Ce6. This was caused by the fluorescence resonance energy transfer process. As predicted, there was an obvious decrease in the whole emission region after MUCNPs were modified onto BPNs, due to the nonspecific absorbency [42,46]. XPS was applied to investigate the chemical state of manganese element in the as-made MUCNPs. The two characteristic peaks at 652.8 and $641.3 \mathrm{eV}$, correspond to the Mn (IV) 2 $\mathrm{p}_{2 / 3}$ and Mn (IV) $2 \mathrm{p}_{1 / 2}$ spin-orbit peaks of $\mathrm{MnO}_{2}$, respectively [47], proving that $\mathrm{Mn}^{2+}$ was oxidized into $\mathrm{MnO}_{2}$ under the high temperature and pressure (Figure S5). Figure $\mathbf{1 K}$ shows the magnetic hysteresis measurements of the superparamagnetic MUCNPs with saturation magnetization value of approximately $53.0 \mathrm{emu} / \mathrm{g}$, and that of MUCNPs@BPNs-Ce6 was approximately 5.2 emu/g at room temperature. The decrease was caused by the thick shell coating on the magnetic NPs. Inset in Figure 1K indicates that the MUCNPs@BPNs-Ce6 exhibited a sufficiently strong magnetic separation for biomedical applications. 
As illustrated in Figure 2A, the MUCNPs@BPNs-Ce6 exhibited high stability in physiological media during 7 days, including in water, PBS, RPMI, and DMEM, due to the modification of PL [48]. The absorbance spectra of the MUCNPs@BPNs-Ce6 in physiological media also demonstrates the fluorescent properties of MUCNPs@BPNs-Ce6 (Figure 2B). The absorbance peak matched well with the emission peak of Ce6 at around $370 \mathrm{~nm}$ and $640 \mathrm{~nm}$. Under continuous laser irradiation at $808 \mathrm{~nm}$ $\left(2.0 \mathrm{~W} / \mathrm{cm}^{2}\right)$, the temperature elevation of the various aqueous suspensions containing MUCNPs@BPNs-Ce6 was measured and MUCNPs@BPNs-Ce6 solutions showed an obvious concentration-dependent heating effect (Figure 2C). The control experiment demonstrated that the temperature of the MUCNPs@BPNs-Ce6 solution increased by $30{ }^{\circ} \mathrm{C}$ from room temperature with a concentration of only $200 \mu \mathrm{g} / \mathrm{mL}$ (Figure 2D). Afterwards, we measured the photothermal conversion efficiency $(\eta)$ of the MUCNPs@BPNs-Ce6. Based on the obtained data (Figure 2E,F), the $\eta$ value of MUCNPs@BPNs-Ce6 was determined to be ca.33.4\%, enabling the use of such nanocomposites as a photothermal agent.

As we know, the $\mathrm{Mn}^{2+}$ can be oxidized to $\mathrm{MnO}_{2}$ under high temperature and pressure. The obtained product could further react with $\mathrm{H}_{2} \mathrm{O}_{2}$ and efficiently induce the catalytic decomposition of $\mathrm{H}_{2} \mathrm{O}_{2}$ to generate $\mathrm{O}_{2}$ [48-50]. To investigate the $\mathrm{O}_{2}$ generation capability of MUCNPs@BPNs-Ce6, the dissolved $\mathrm{O}_{2}$ in MUCNPs@BPNs-Ce6 solution was measured with an $\mathrm{O}_{2}$ probe. As shown in Figure 2G, $\mathrm{O}_{2}$ was generated only when the MUCNPs@BPNs-Ce6 and $\mathrm{H}_{2} \mathrm{O}_{2}$ coexisted, and no $\mathrm{O}_{2}$ was detected in pure $\mathrm{H}_{2} \mathrm{O}_{2}$, MUCNPs@BPNs-Ce6/water or MUCNPs@BPNs-Ce6/PBS. Since the MUCNPs@BPNs-Ce6 could effectively generate $\mathrm{O}_{2}$ from $\mathrm{H}_{2} \mathrm{O}_{2}$, the electron spin resonance (ESR) technique was performed to measure the ${ }^{1} \mathrm{O}_{2}$-generation capability of the MUCNPs@BPNs-Ce6 that was dispersed in acidic $\mathrm{H}_{2} \mathrm{O}_{2}$ with 2,2,6,6-tetramethylpiperidine-N-oxyl (TEMPO) as an ${ }^{1} \mathrm{O}_{2}$ trapper. As shown in Figure $\mathbf{2 H}$, a characteristic spin adduct with three resolved peaks was found, which revealed that ${ }^{1} \mathrm{O}_{2}$ were generated from MUCNPs@BPNs-Ce6 upon NIR light irradiation. Besides, 1,3diphenylisobenzofuran (DPBF) could react with $\mathrm{H}_{2} \mathrm{O}_{2}$ and cause an irreversible decrease in the 
absorbance of DPBF at $416 \mathrm{~nm}$, it was applied as the trapping agent to assess the ${ }^{1} \mathrm{O}_{2}$ quantum yield of the MUCNPs@BPNs-Ce6 (Figure 2I). Under 808 nm laser $\left(0.1 \mathrm{~W} / \mathrm{cm}^{2}\right)$ irradiation, the absorbance of DPBF decreased gradually during the irradiation with the presence of the MUCNPs@BPNs-Ce6, indicating that MUCNPs@BPNs-Ce6 could generate enough ROS and be potentially utilized in PDT.
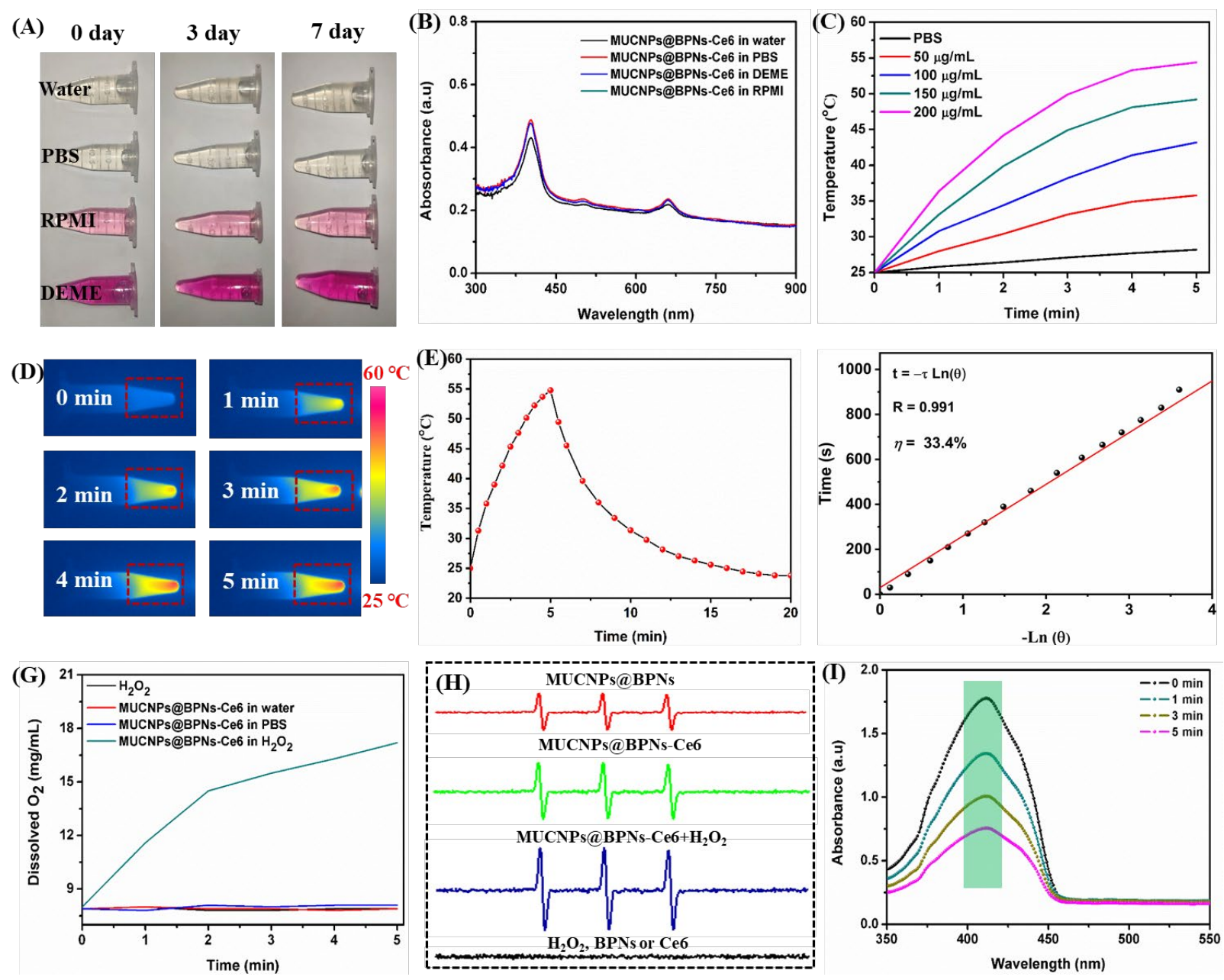

Figure 2. (A) The dispersed stability of the MUCNPs@BPNs-Ce6 in physiological mediums. (B) Absorbance spectra of the MUCNPs@BPNs-Ce6 in physiological mediums. (C) The photothermal heating curves of PBS and MUCNPs@BPNs-Ce6 solutions with different concentrations under the $808 \mathrm{~nm}$ laser irradiation $(2.0$ $\mathrm{W} / \mathrm{cm}^{2}$ ). (D) Temperature infrared images of $200 \mu \mathrm{g} / \mathrm{mL}$ MUCNPs@BPNs-Ce6 aqueous solution under $808 \mathrm{~nm}$ laser irradiation $\left(2.0 \mathrm{~W} / \mathrm{cm}^{2}\right)$ irradiation. (E) Photothermal response of MUCNPs@BPNs-Ce6 aqueous solution under irradiation for $5 \mathrm{~min}$ under $808 \mathrm{~nm}$ laser irradiation $\left(2.0 \mathrm{~W} / \mathrm{cm}^{2}\right)$ and then the laser was shut off. (F) Linear time data versus - $\mathrm{Ln} \theta$ obtained from the heating up time of $(\mathrm{E})$. (G) The $\mathrm{O}_{2}$ concentration in $\mathrm{H}_{2} \mathrm{O}_{2}$ solutions and different solutions after incubation with MUCNPs@BPNs-Ce6. (H) ESR spectra of the MUCNPs@BPNs-Ce6 in different reaction systems. (I) Absorbance spectra of DPBF mixed with the MUCNPs@BPNs-Ce6.

The biocompatibility of MUCNPs@BPNs-Ce6 was also investigated through erythrocyte shapes, hemolysis assay, and methyl thiazolyl tetrazolium (MTT) assay. Morphologically aberrant forms of 
erythrocytes could be applied to the diagnosis of various medical conditions, including hemolytic anemia. The photomicrographs from Figure 3A indicates that the number and shape of erythrocytes in the test blood did not change after the treatment of MUCNPs@BPNs-Ce6. As shown in Figure 3B, no significant hemolysis was observed when red blood cells (RBCs) were co-cultured with MUCNPs@BPNs-Ce6. It was found that MUCNPs@BPNs-Ce6 alone had no toxic effect on HeLa cells, even though their concentrations were up to $500 \mu \mathrm{g} / \mathrm{mL}$ (Figure 3C). Besides, morphology change and cell apoptosis of HeLa cells were hardly observed under optical microscope from morphology imaging of cells (Figure 3D). These results suggested that MUCNPs@BPNs-Ce6 had insignificant cytotoxicity and possessed good biocompatibility in vitro [28,51].
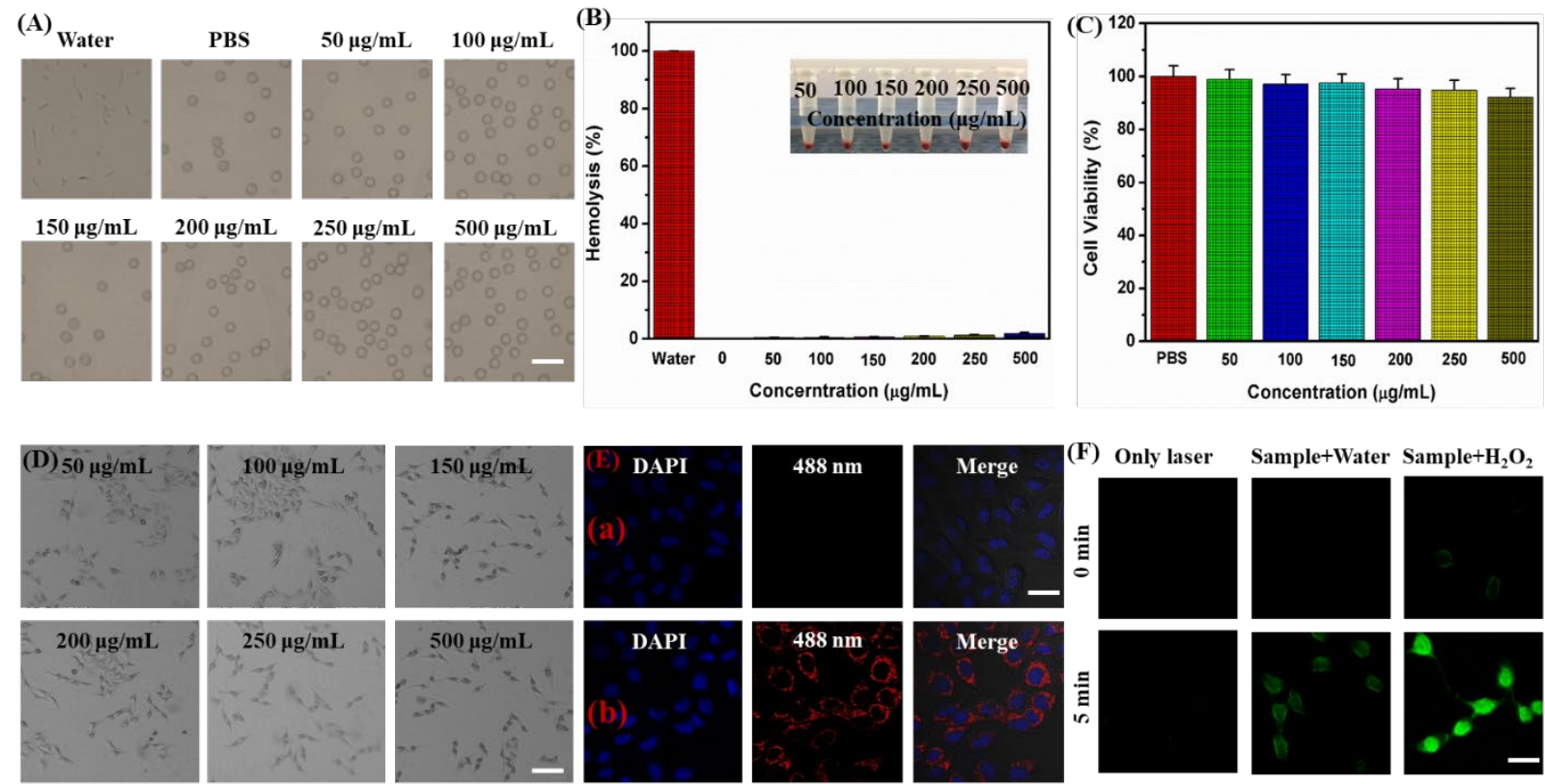

F) Only laser Sample+Water Sample $+\mathrm{H}_{2} \mathrm{O}_{2}$
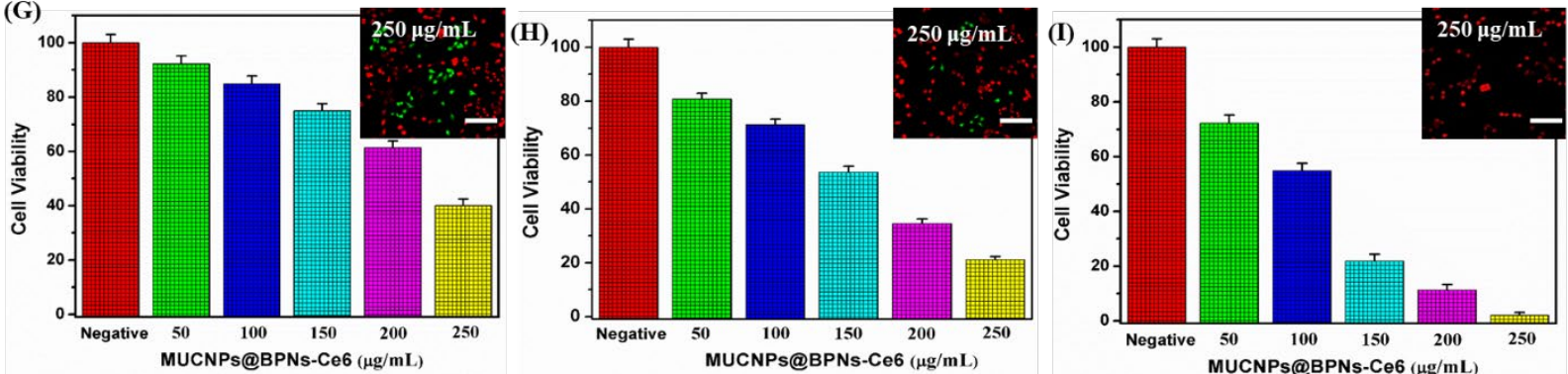

Figure 3. (A) Erythrocyte shapes of the rat after injection of water, PBS and MUCNPs@BPNs-Ce6. Scale bar: $50 \mu \mathrm{m}$. (B) Hemolysis percentage of RBCs at various concentrations of MUCNPs@BPNs-Ce6. Inset is the photograph of the RBCs solution cultured with MUCNPs@BPNs-Ce6. (C) Cell viability of HeLa cells with various concentrations of MUCNPs@BPNs-Ce6. (D) Morphology of HeLa cells incubated with various concentrations of MUCNPs@BPNs-Ce6. Scale bar: $100 \mu \mathrm{m}$. (E) Confocal fluorescence images of HeLa cells 
incubated with (a) PBS or (b) MUCNPs@BPNs-Ce6 for 4 h. Scale bar: $30 \mu \mathrm{m}$. (F) Fluorescence images of SOSG stained HeLa cells in a $\mathrm{N}_{2}$ atmosphere. Scale bar: $50 \mu \mathrm{m}$. Cell viability of HeLa cells with various concentrations of MUCNPs@BPNs-Ce6 under different conditions: $(\mathrm{G}) 0.1 \mathrm{~W} / \mathrm{cm}^{2}+$ acid $\mathrm{H}_{2} \mathrm{O}_{2}$ solution; $(\mathrm{H})$ $2.0 \mathrm{~W} / \mathrm{cm}^{2}$; (I) $2.0 \mathrm{~W} / \mathrm{cm}^{2}+$ acid $\mathrm{H}_{2} \mathrm{O}_{2}$ solution for $5 \mathrm{~min}$. Inset is the confocal fluorescence images of calcein AM/PI-stained HeLa cells incubated with various treatments. Scale bar: $100 \mu \mathrm{m}$.

The cellular uptake behaviors of MUCNPs@BPNs-Ce6 inside HeLa cells could be guided by the Ce6 fluorescence. Red fluorescence came from Ce6 and blue fluorescence in cell nucleus came from 4',6-diamidino-2-phenylindole (DAPI). As shown in Figure 3E, the cytoplasm of HeLa cells emitted strong red fluorescence, and no red fluorescence signal was observed in cell nucleus, demonstrating that MUCNPs@BPNs-Ce6 had entered into cells but had been hampered by the numerous small nuclear pore complexes of the nuclear membrane [52-54]. In addition, a singlet oxygen sensor green (SOSG) probe was used to measure the intracellular ${ }^{1} \mathrm{O}_{2}$ generation of the MUCNPs@BPNs-Ce6 assembly, which could react with ${ }^{1} \mathrm{O}_{2}$ from PDT and generate an endoperoxide product with green fluorescence. To protect the cellular morphology, 5\% paraformaldehyde was used to immobilize HeLa cells and were incubated with the MUCNPs@BPNs-Ce6. Then, the fixed cells were washed with PBS and further incubated with SOSG for $5 \mathrm{~min}$. When the fixed cells were irradiated with an $808 \mathrm{~nm}$ laser for 5 min, only weak green fluorescence of SOSG was observed (Figure 3F) in the HeLa cells. In contrast, the cells showed enhanced fluorescence once the cells were mixed with $\mathrm{H}_{2} \mathrm{O}_{2}$ and subsequently irradiated by 808 nm laser, indicating that the MUCNPs@BPNs-Ce6 could efficiently generate intracellular ${ }^{1} \mathrm{O}_{2}$ for PDT.

To evaluate the therapeutic effect of our nanocarriers, a live/dead assay and MTT assay were carried out by incubating cancer cells with MUCNPs@BPNs-Ce6, then the cells were illuminated with different NIR laser. After proper staining, a control experiment, where cancer cells were incubated without nanocarriers but exposed to the laser irradiation, showed high cell viability, indicating the irradiation alone had no adverse impact on cell survival $[55,56]$. However, it was observed that MUCNPs@BPNs-Ce6 treated cells under NIR light showed obvious viabilities of reductions (Figure 3G), indicating that MUCNPs@BPNs-Ce6 had a good PDT ability. In addition, a lower cell viability 
was found when MUCNPs@BPNs-Ce6 was used under increasing laser power intensities (Figure 3H). Interestingly, the cell viability further decreased when MUCNPs@BPNs-Ce6 with $\mathrm{H}_{2} \mathrm{O}_{2}$ solution was applied along with laser irradiation $\left(2.0 \mathrm{~W} / \mathrm{cm}^{2}\right)$, indicating that the cell death was not only PTTdependent, but also due to a synergetic effect coming from the MUCNPs@BPNs-Ce6 (Figure 3I). As expectant, this synergetic effect could be clearly testified by $250 \mu \mathrm{g} / \mathrm{mL}$ of MUCNPs@BPNs-Ce6 under laser irradiation, resulting in almost $100 \%$ cell death. Confocal fluorescence images of calcein AM and propidium iodide (PI) contained cells further confirmed the efficiency of photothermal and photodynamic ablation induced by MUCNPs@BPNs-Ce6 (inset of Figure 3I).

As mentioned above, the obtained $\mathrm{MnO}_{2}$ could decompose into $\mathrm{Mn}^{2+}$ at a reduced $\mathrm{pH}$ in tumor microenvironment. It has been known that the $\mathrm{Mn}^{2+}$ and $\mathrm{Fe}_{3} \mathrm{O}_{4}$ have been widely applied as contrast agents for MR imaging. Therefore, the obtained MUCNPs@BPNs-Ce6 showed an obvious concentration-dependent brightening effect under T1-weighted MR imaging, as well as darkening effect under T2-weighted MR imaging. Figure 4A,B displays different relaxation time (T1 and T2) of MUCNPs@BPNs-Ce6 with different concentrations. The T1 and T2 longitudinal relaxivity of MUCNPs@BPNs-Ce6 ( $\mathrm{r} 1$ and $\mathrm{r} 2$ ) was measured to be $9.79 \mathrm{mM}^{-1} \mathrm{~s}^{-1}$ and $5.71 \mathrm{mM}^{-1} \mathrm{~s}^{-1}$, which was linearly enhanced along with the increase of equivalent $\mathrm{Mn}^{2+}$ and $\mathrm{Fe}$ concentrations. The blood circulation and biodistribution profiles are important to study the in vivo behavior of nanocomposites. Uptake of MUCNPs@BPNs-Ce6 in blood was investigated by detecting Fe concentration, and it decreased to 4\% ID/g at $24 \mathrm{~h}$ in MUCNPs@BPNs-Ce6 injected mice, and remained at a relatively high level for a long time (Figure 4C). As shown in Figure 4D, a clear magnetic resonance response from in vivo T1 MR imaging was observed at the tumor site at $24 \mathrm{~h}$ post injection, owing to the gradual generation of $\mathrm{Mn}^{2+}$ from the $\mathrm{MnO}_{2}$ of MUCNPs. In addition, the quantitative MR imaging of the quantification of the region-of-interest (ROI) also verified higher T1-weighted MR intensity, further proving the effective tumor accumulation of nanocomposites (Figure 4E). Meanwhile, reduced T2weighted MR signals in the tumor showed up, and the signals stayed at an almost constant level after 
intravenous injection from the in vivo T2 MR imaging (Figure 4D, and Figure S7). These results proved that MUCNPs@BPNs-Ce6 were a good application prospect in magnetic resonance imaging in vivo and could serve as a $\mathrm{T} 1$ and $\mathrm{T} 2$ contrast agent simultaneously.
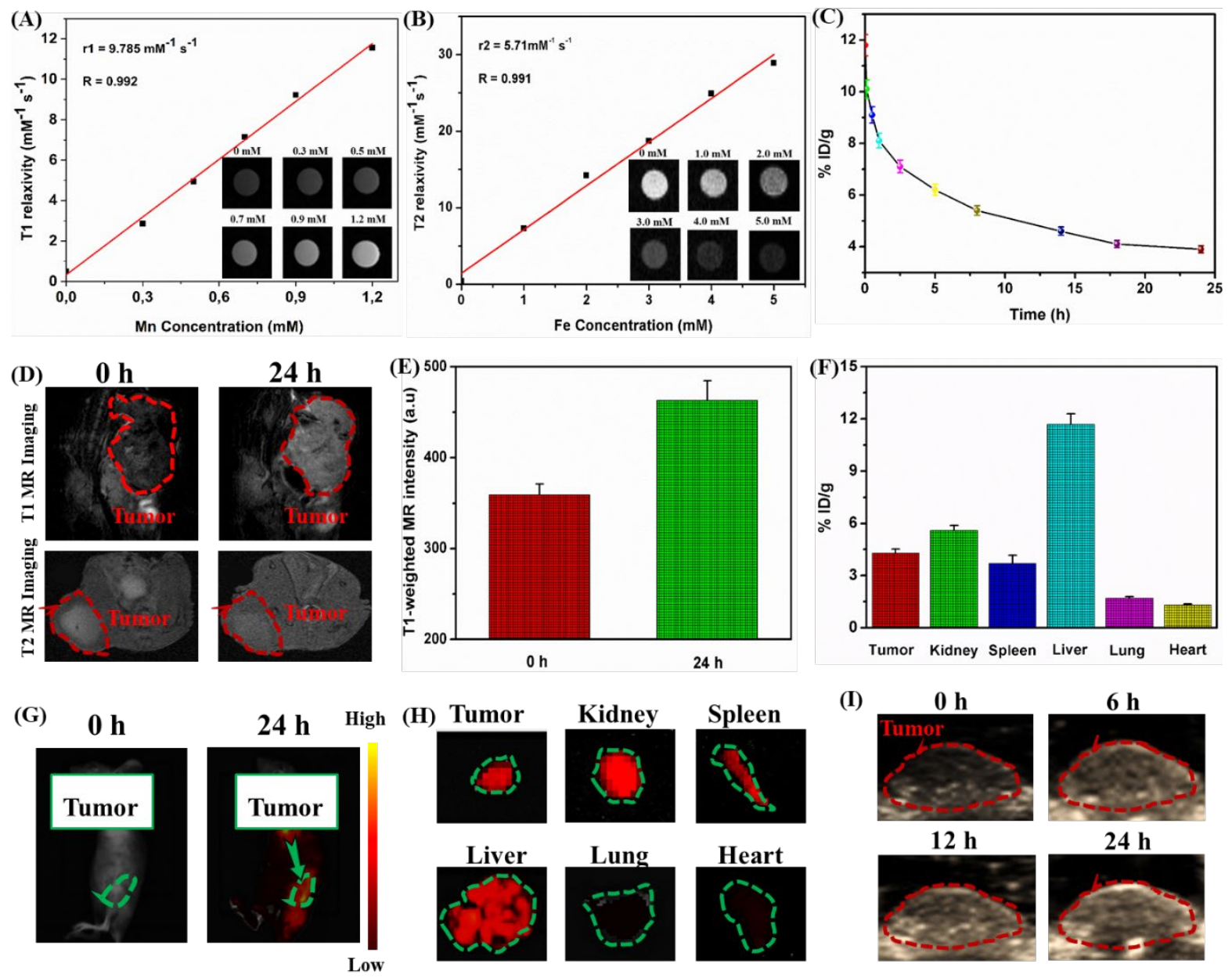

(I)
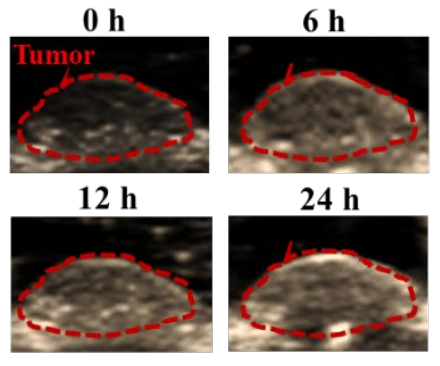

Figure 4. (A) Linear correlation between longitudinal relaxivities (r1) and equivalent Mn concentration of MUCNPs@BPNs-Ce6 at pH 6. The inset shows T1-weighted MR images of MUCNPs@BPNs-Ce6 solution with various Mn concentrations at pH 6. (B) Linear correlation between longitudinal relaxivities (r2) and equivalent Fe concentration of MUCNPs@BPNs-Ce6. The inset shows T2-weighted MR images of MUCNPs@BPNs-Ce6 solution with various Fe concentrations. (C) Blood time-activity curve for MUCNPs@BPNs-Ce6 by measuring Fe concentration. (D) In vivo MR images of the tumor of mice before and after injection with MUCNPs@BPNs-Ce6 for $24 \mathrm{~h}$. (E) T1-weighted MR signals in the tumor before and after injection with MUCNPs@BPNs-Ce6 for 24 h. (F) Biodistribution results of HeLa tumor-bearing mice before and after injection with MUCNPs@BPNs-Ce6 in MR imaging for 24 h. (G) In vivo fluorescence images of HeLa tumor-bearing mice before and after injection with MUCNPs@BPNs-Ce6 for 24 h. (H) Ex vivo fluorescence images of organs and tumor after injection with MUCNPs@BPNs-Ce6 for 24 h. (I) In vivo US imaging of HeLa tumor-bearing mice after injection with MUCNPs@BPNs-Ce6 for different time.

Analysis of radioactivity in isolated organs and tissues injected with MUCNPs@BPNs-Ce6 for $24 \mathrm{~h}$ could be observed in Figure 4F. A considerable signal of Fe $(>10 \% \mathrm{ID} / \mathrm{g})$ in the liver organs was measured after the injection of MUCNPs@BPNs-Ce6, and the signal was lowest in the heart which 
was around $1.5 \% \mathrm{ID} / \mathrm{g}$. Significant differences in blood retention and tumor uptake became evident in the image analysis depending on the nanocomposites surface modification. Figure 4G shows the in vivo fluorescence imaging of HeLa tumor-bearing mice injected with MUCNPs@BPNs-Ce6. Compared with the image prior to injection, a strong signal could be observed in the tumor region of HeLa tumor-bearing mice injected with MUCNPs@BPNs-Ce6 for 24 h, indicating the efficient accumulation of those nanocomposites in the tumor. In vivo fluorescence imaging of isolated organs further confirmed that nanocomposites accumulated mainly in the liver and almost no fluorescence signal was found in the heart (Figure $\mathbf{4 H}$ and Figure S8). Furthermore, it was obviously through observation that the ultrasound signals appeared at the tumor site after intravenous injection, which was attributed to $\mathrm{O}_{2}$ bubbles coming from the decomposition of $\mathrm{H}_{2} \mathrm{O}_{2}$ (Figure 4I).

In vivo exploration was carried out in four groups of HeLa tumor-bearing nude mice $(\mathrm{n}=3)$ to test the therapeutic efficacy of MUCNPs@BPNs-Ce6 via the tail vein (Figure 5A). The tumor temperature under irradiation was monitored by the thermal camera. Five minutes after the irradiation, tumor temperature increased to higher than $50{ }^{\circ} \mathrm{C}$ (Figure 5B) in MUCNPs@BPNs-Ce6 with 808 nm laser $\left(2.0 \mathrm{~W} / \mathrm{cm}^{2}\right)$ treated groups, which was sufficient to cause tumor cells damage in vivo. By comparison, for mice treated with PBS and low NIR light exposure, tumor temperature did not show any obvious increase. These results suggested that light energy can transfer into thermal energy efficiently in the body by MUCNPs@BPNs-Ce6. Figure 5C shows the therapeutic effects of different groups. The tumor-bearing nude mice were treated as follows: group 1, PBS (control); group 2, PBS irradiated with 808 nm laser (2.0 W/cm²); group 3, MUCNPs@BPNs-Ce6 irradiated with 808 nm laser $\left(0.1 \mathrm{~W} / \mathrm{cm}^{2}\right)$; and group 4, MUCNPs@BPNs-Ce6 irradiated with 808 nm laser $\left(2.0 \mathrm{~W} / \mathrm{cm}^{2}\right)$. The tumor volumes were calculated every 2 days. At the end of the experiment, all nude mice were put down and the tumors were collected. The tumor sizes of the MUCNPs@BPNs-Ce6 (Figure 5D,E) under 808 nm laser $\left(2.0 \mathrm{~W} / \mathrm{cm}^{2}\right)$ irradiation group were notably smaller than those of the other three groups. Consequently, these results demonstrated that the laser irradiation-controlled MUCNPs@BPNs-Ce6 
possessed an excellent tumor ablation effect in vivo. In mice treated with MUCNPs@BPNs-Ce6 followed by laser irradiation and applied magnetic field, the tumor tissues were necrotic, which exhibited karyolysis, pyknosis, and degradation (Figure 5F). By contrast, the tumor tissues of the PBS group exhibited a normal organized cellular structure.

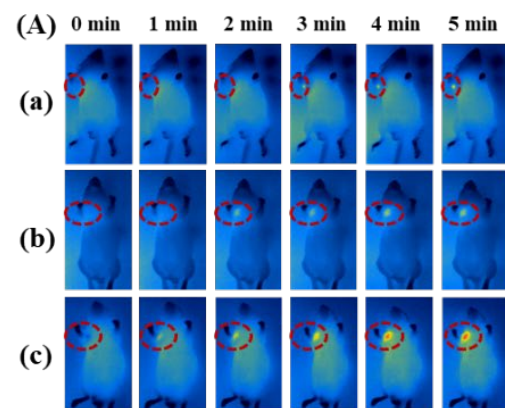

(D)
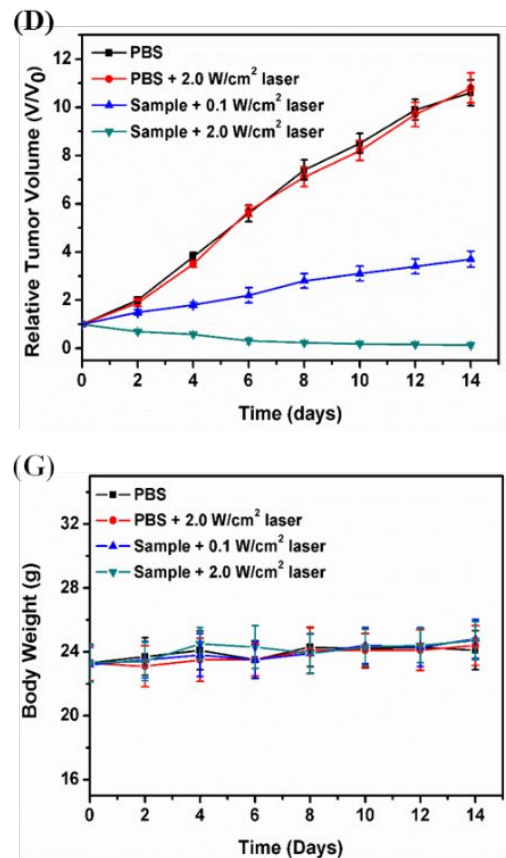

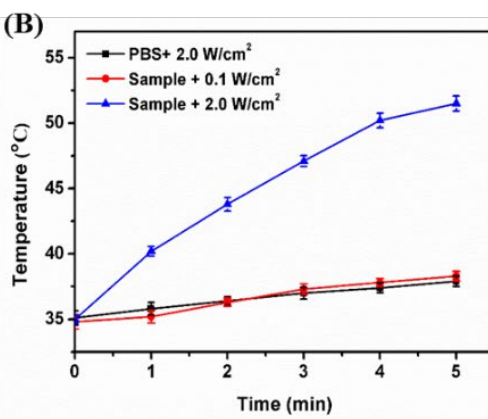

(E)

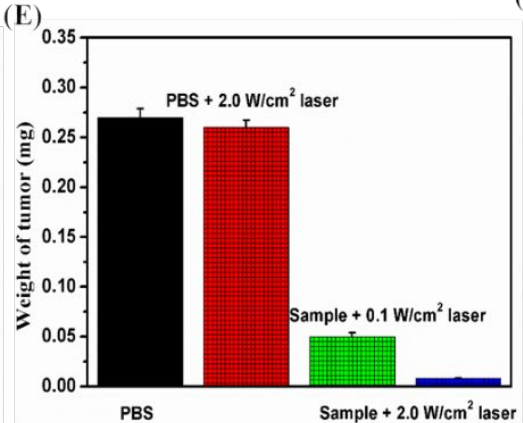

(C)

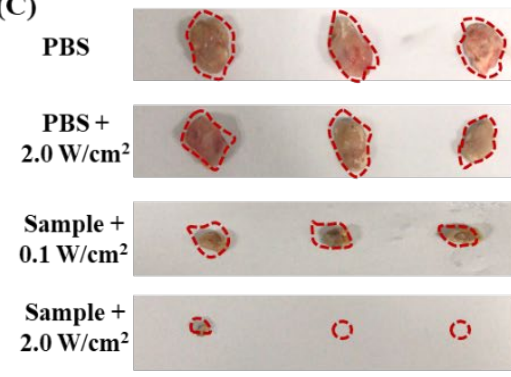

(F)
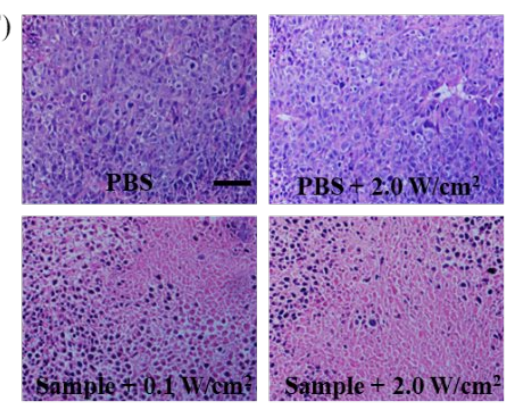

(I)

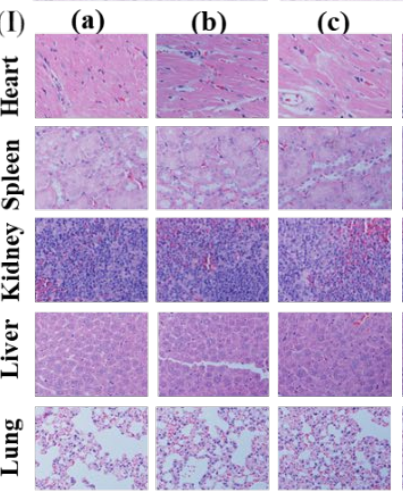

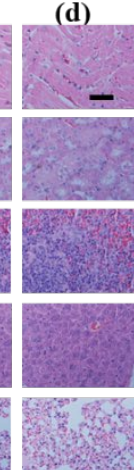

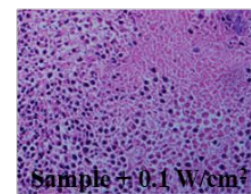

(b)

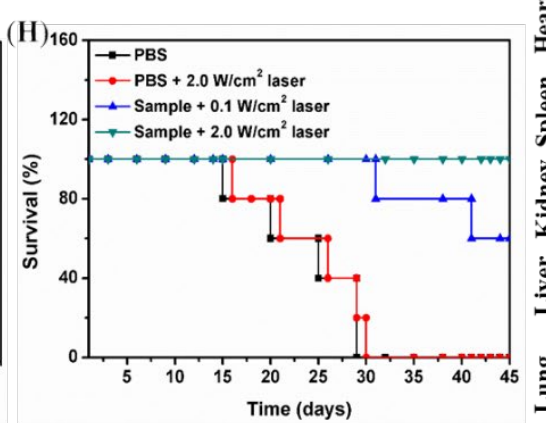

Figure 5. (A) Thermal images of tumor-bearing mice exposed to the $808 \mathrm{~nm}$ laser irradiation for 5 min. (B) Temperature changes of tumors after $808 \mathrm{~nm}$ laser irradiation. (C) Photographs of excised tumors after different treatment. (D) In vivo tumor volume curves of tumor-bearing mice in different groups after various treatments indicated. (E) The weight of excised tumor. (F) Hematoxylin and eosin (H\&E) staining photomicrographs of tumor tissue after different treatments. Size bar $=100 \mu \mathrm{m}$. (G) Body weights after different treatments. (H) Percent survival after different treatment groups. (I) Histopathological analysis of major organs after different treatments: (a) PBS; (b) $\mathrm{PBS}+2.0 \mathrm{~W} / \mathrm{cm}^{2}$ laser; (c) sample $+0.1 \mathrm{~W} / \mathrm{cm}^{2}$ laser; and (d) sample $+2.0 \mathrm{~W} / \mathrm{cm}^{2}$ laser. Size bar $=100 \mu \mathrm{m}$.

Furthermore, the body weight of mice was not significantly affected, demonstrating that there were no acute side effects along with our combined therapy (Figure 5G). Noticeably, the MUCNPs@BPNs-Ce6 with $2.0 \mathrm{~W} / \mathrm{cm}^{2}$ treated group showed a 100\% survival rate after 45 days. In contrast, mice of other treated groups had lower survival or even died owing to the extensive tumor 
burden (Figure 5H). The in vivo biosafety study showed that MUCNPs@BPNs-Ce6 caused no damage to the main organs (Figure 5I). It is well known that most of the intravenously injected nanocomposites are taken up and eliminated by mononuclear phagocyte system (MPS), including liver and kidney tissue [57]. Therefore, acute inflammation in the liver and kidney caused by MUCNPs@BPNs-Ce6 could be characterized by an increase in biochemical parameters, including alanine aminotransferase (ALT), aspartate aminotransferase (AST), blood urea nitrogen (BUN), and increased total bilirubin (TBIL). As shown in Figure S9, the biochemical examination of the serum shows that the ALT, AST, BUN, and TBIL levels in the test group had no obvious difference compared to that in the control group. These results indicate that the MUCNPs@BPNs-Ce6 had no noticeable acute toxicity in small experimental animals.

\section{Conclusion}

In summary, a BPNs nanocarrier was designed and prepared to load the MUCNPs and Ce6 through chemical crosslinking, for tumor phototherapy guided by multimodal imaging. The obtained MUCNPs@BPNs-Ce6 demonstrated excellent light conversion efficiency, biocompatibility, and imaging properties. Through enhanced permeability and retention (EPR) effect, the nanocarriers could efficiently aggregate at the site of the tumor, which effectively improved therapeutic effects and enhanced imaging. Upon irradiation at 808 nm NIR light, the MUCNPs@BPNs-Ce6 possessed efficient ROS generation and high photothermal conversion efficiency, benefiting from PTT/PDT synergistic therapy of BPNs and $\mathrm{Ce} 6$, as well as a higher $\mathrm{O}_{2}$ supply for $\mathrm{MnO}_{2}$. Meanwhile, the multimodality imaging of MUCNPs@BPNs-Ce6 could be applied for diagnosis. Both in vitro and in vivo experiments verified the safety and enhanced antitumor effect of this nanoplatform. Taken together, the current work demonstrated that MUCNPs@BPNs-Ce6 can be exploited as a good theranostic agent for cancer diagnosis and treatment. 


\section{Acknowledgement}

This work was supported by the Jiangsu six category outstanding talent (2012-NY-031), Jiangsu province science and technology support plan (BE2015367), and the Villum Fonden, Denmark, Project No. 13153. B.S., M.Z., and T.Z. would like to thank the CSC for its generous support. We would like to thank Dr. Jon Ashley for helping with the manuscript proof-reading.

\section{References}

[1] J. Wu, J. Tian, L. Rui, W. Zhang, Enhancing the efficacy of photodynamic therapy (PDT) via water-soluble pillar [5] arene-based supramolecular complexes, Chem. Commun. 54 (2018) 7629-7632.

[2] J.R. Choi, K.W. Yong, J.Y. Choi, A. Nilghaz, Y. Lin, J. Xu, X.N. Lu, Black phosphorus and its biomedical applications, Theranostics, 8 (2018) 1005-1026.

[3] A. Lin, Y. Sun, H. Zhang, X. Lin, L. Yang, Q. zheng, Fluctuating characteristics of air-mist mixture flow with conjugate wall-film motion in a compressor of gas turbine, Appl. Therm. Eng. 142 (2018) 779-792.

[4] Y. Ding, J. Wan, Z. Zhang, F. Wang, J. Guo, C. Wang, Localized Fe (II)-induced cytotoxic reactive oxygen species generating nanosystem for enhanced anticancer therapy, ACS Appl. Mater. Interfaces 10 (2018) 4439-4449.

[5] W. Zhang, J. Lu, X. ao, P. Li, W. Zhang, Y. Ma, B. Tang, Enhanced photodynamic therapy by reduced levels of intracellular glutathione obtained by employing a nano-MOF with $\mathrm{Cu}$ II as the active center, Angew. Chem., Int. Ed. 57 (2018) 4891-4896.

[6] L.H. Liu, W.X. Qiu, Y.H. Zhang, B. Li, C. Zhang, F. Gao, X.Z. Zhang, A charge reversible selfdelivery chimeric peptide with cell membrane-targeting properties for enhanced photodynamic therapy, Adv. Funct. Mater. 27 (2017) 1700220.

[7] T. Zheng, W. Wang, F. Wu, M. Zhang, J. Shen, Y. Sun, Zwitterionic polymer-gated Au@ $\mathrm{TiO}_{2}$ core-shell nanoparticles for imaging-guided combined cancer therapy, Theranostics 9 (2019) 5035-5048.

[8] S. Anju, J. Ashtami, P.V. Mohanan, Black phosphorus, a prospective graphene substitute for biomedical applications, Mater. Sci. Eng. C 97 (2019) 978-993.

[9] M. Zhang, W.T. Wang, F. Wu, K. Graveran, J. Zhang, C.Z. Wu, Black phosphorus quantum dots gated, carbon-coated $\mathrm{Fe}_{3} \mathrm{O}_{4}$ nanocapsules (BPQDs@ss- $\mathrm{Fe}_{3} \mathrm{O}_{4} @ \mathrm{C}$ ) with low premature release could enable imaging-guided cancer combination therapy, Chem. Eur. J. 24 (2018) 12890-12901.

[10] L. Bai, X. Wang, S. Tang, Y. Kang, J. Wang, Y. Yu, P.K. Chu, Black phosphorus/platinum heterostructure: a highly efficient photocatalyst for solar-driven chemical reactions, Adv. Mater. 30 (2018) 1803641.

[11] M. Tatullo, F. Genovese, E. Aiello, M. Amantea, I. Makeeva, B. Zavan, S. Rengo, L. Fortunato, Phosphorene Is the New Graphene in Biomedical Application, Materials 12 (2019) 2301.

[12] S.J. Kim, H.J. Koh, C.E. Ren, O. Kwon, K. Maleski, S.Y. Cho, Y. Gogotsi, Metallic $\mathrm{Ti}_{3} \mathrm{C}_{2} \mathrm{~T}_{\mathrm{x}}$ MXene gas sensors with ultrahigh signal-to-noise ratio, ACS nano 12 (2018) 986-993.

[13] M. Qiu, A. Singh, D. Wang, J. Qu, M. Swihart, H. Zhang, P.N. Prasad, Biocompatible and 
biodegradable inorganic nanostructures for nanomedicine: Silicon and black phosphorus, Nano Today 25 (2019) $135-155$

[14] S. Wang, J. Lin, T. Wang, X. Chen, P. Huang, Recent advances in photoacoustic imaging for deep-tissue biomedical applications, Theranostics 6 (2016) 2394-2413.

[15] Y. Zheng, Z.H. Yu, H.H. Ou, A. M. Asiri, Y.L. Chen, X.C. Wang, Black phosphorus and polymeric carbon nitride heterostructure for photoinduced molecular oxygen activation, Adv. Funct. Mater. 28 (2018) 1705407.

[16] Z. Li, H. Xu, J. Shao, C. Jiang, F. Zhang, J. Lin, P. Huang, Polydopamine-functionalized black phosphorus quantum dots for cancer theranostics, Appl. Mater. Today 15 (2019) 297-304.

[17] M. Caporali, M. Serrano-Ruiz, F. Telesio, S. Heun, G. Nicotra, C. Spinella, M. Peruzzini, Decoration of exfoliated black phosphorus with nickel nanoparticles and its application in catalysis, Chem. Commun. 53 (2017) 10946-10949.

[18] Y. Li, Z. Liu, Y. Hou, G. Yang, X. Fei, H. Zhao, Z. Zhuang, Multifunctional nanoplatform based on black phosphorus quantum dots for bioimaging and photodynamic/photothermal synergistic cancer therapy, ACS Appl. Mater. Interfaces 9 (2017) 25098-25106.

[19] R. Tian, S. Zhao, G. Liu, H. Chen, L. Ma, H. You, Z. Wang, Construction of lanthanide-doped upconversion nanoparticle-Uelx Europaeus Agglutinin-I bioconjugates with brightness red emission for ultrasensitive in vivo imaging of colorectal tumor, Biomaterials 212 (2019) 64-72.

[20] Q. Guo, A. Pospischil, M. Bhuiyan, H. Jiang, H. Tian, D. Farmer, B. Deng, C. Li, S. Han, H. Wang, Q. Xia, T. Ma, T. Mueller, F. Xia, Black phosphorus mid-infrared photodetectors with high gain, Nano Lett. 16 (2016) 4648-4655.

[21] J. Liu, P. Du, H. Mao, L. Zhang, H. Ju, J. Lei, Dual-triggered oxygen self-supply black phosphorus nanosystem for enhanced photodynamic therapy, Biomaterials 172 (2018) 83-91.

[22] T. Guo, Y. Lin, G. Jin, R. Weng, J. Song, X. Liu, H. Yang, Manganese-phenolic network-coated black phosphorus nanosheets for theranostics combining magnetic resonance/photoacoustic dual-modal imaging and photothermal therapy, Chem. Commun. 55 (2019) 850-853.

[23] J. Shen, G. Chen, A.M. Vu, W. Fan, O.S. Bilsel, C.C. Chang, G. Han, Engineering the upconversion nanoparticle excitation wavelength: cascade sensitization of tri-doped upconversion colloidal nanoparticles at $800 \mathrm{~nm}$, Adv. Opt. Mater. 1 (2013) 644-650.

[24] Y. You, D. Xu, X. Pan, X. Ma, Self-propelled enzymatic nanomotors for enhancing synergetic photodynamic and starvation therapy by self-accelerated cascade reactions, Appl. Mater. Today 16 (2019) 508517.

[25] Y.C. Tsai, P. Vijayaraghavan, W.H. Chiang, H.H. Chen, T.I. Liu, M.Y. Shen, H.C. Chiu, Targeted delivery of functionalized upconversion nanoparticles for externally triggered photothermal/photodynamic therapies of brain glioblastoma, Theranostics 8 (2018) 1435.

[26] L. Feng, F. He, B. Liu, G. Yang, S. Gai, P. Yang, J. Lin, g-C $\mathrm{C}_{3} \mathrm{~N}_{4}$ coated upconversion nanoparticles for $808 \mathrm{~nm}$ near-infrared light triggered phototherapy and multiple imaging, Chem. Mater. 28 (2016) 7935-7946.

[27] T. Zheng, T. Zhou, X. Feng, J. Shen, M. Zhang, Y. Sun, Enhanced plasmon-induced resonance energy transfer (PIRET)-mediated photothermal and photodynamic therapy guided by photoacoustic and magnetic resonance imaging, ACS Appl. Mater. Interfaces 11 (2019) 31615-31626.

[28] R. Lv, D. Yang, P. Yang, J. Xu, F. He, S. Gai, J. Lin, Integration of upconversion nanoparticles and ultrathin black phosphorus for efficient photodynamic theranostics under $808 \mathrm{~nm}$ near-infrared light irradiation, Chem. Mater. 28 (2016) 4724-4734.

M.L. You, J. Zhong, Y. Hong, Z.F. Duan, M. Lin, F. Xu, Inkjet printing of upconversion 
nanoparticles for anti-counterfeit applications, Nanoscale 7 (2015) 4423-4431.

[30] G.A. Mandl, P.A. Rojas-Gutierrez, J.A. Capobianco, A NIR-responsive azobenzene-based supramolecular hydrogel using upconverting nanoparticles, Chem. Commun. 54 (2018) 5847-5850.

[31] Y. Zhao, Y.H. Zhang, Z. Zhuge, Y.H. Tang, J.W. Tao, Y. Chen, Synthesis of a poly-L-lysine/black phosphorus hybrid for biosensors, Anal. Chem. 90 (2018) 3149-3155.

[32] Z. Yu, W. Pan, N. Li, B. Tang, A nuclear targeted dual-photosensitizer for drug-resistant cancer therapy with NIR activated multiple ROS, Chem. Sci. 7 (2016) 4237-4244.

[33] B. Poinard, S.Z.Y. Neo, E.L.L. Yeo, H.P.S. Heng, K.G. Neoh, J.C.Y. Kah, Polydopamine nanoparticles enhance drug release for combined photodynamic and photothermal therapy, ACS Appl. Mater. Interfaces 10 (2018) 21125-21136.

[34] J.P. Wei, J.C. Li, D. Sun, Q. Li, J.Y. Ma, X.L. Chen, X. Zhu, N.F. Zheng, A novel theranostic nanoplatform based on Pd@Pt-PEG-Ce6 for enhanced photodynamic therapy by modulating tumor hypoxia microenvironment, Adv. Funct. Mater. 28 (2018) 1706310.

[35] L. Feng, D. Tao, Z. Dong, Q. Chen, Y. Chao, Z. Liu, M. Chen, Near-infrared light activation of quenched liposomal Ce6 for synergistic cancer phototherapy with effective skin protection, Biomaterials 127 (2017) 13-24.

[36] D.V. Bax, N. Davidenko, D. Gullberg, S.W. Hamaia, R.W. Farndale, S.M. Best, R.E. Cameron, Fundamental insight into the effect of carbodiimide crosslinking on cellular recognition of collagen-based scaffolds, Acta Biomater. 49 (2017) 218-234.

[37] C. Yue, C. Zhang, G. Alfranca, Y. Yang, X. Jiang, D. Cui, Near-infrared light triggered ROSactivated theranostic platform based on Ce6-CPT-UCNPs for simultaneous fluorescence imaging and chemophotodynamic combined therapy, Theranostics 6 (2016) 456-469.

[38] G. Yang, R. Zhang, C. Liang, H. Zhao, X. Yi, S. Shen, Z. Liu, Manganese dioxide coated $\mathrm{WS}_{2} @ \mathrm{Fe}_{3} \mathrm{O}_{4} / \mathrm{sSiO}_{2}$ nanocomposites for pH-responsive MR imaging and oxygen-elevated synergetic therapy, Small 14 (2018) 1702664.

[39] M. Zhang, W.T. Wang, N.L. Zhou, P. Yuan, Y.T. Su, M.N. Shao, C. Chi, F.Y. Pan, Near-infrared light triggered photo-therapy, in combination with chemotherapy using magnetofluorescent carbon quantum dots for effective cancer treating, Carbon 118 (2017) 752-764.

[40] Y.K. Peng, C.N.P. Lui, Y.W. Chen, S.W. Chou, E. Raine, P.T. Chou, K.K.L. Yung, S.C.E. Tsang, Engineering of single magnetic particle carrier for living brain cell imaging: A tunable T1-/T2-/dual-modal contrast agent for magnetic resonance imaging application, Chem. Mater. 29 (2017) 4411-4417.

[41] M. Zhang, W. Wang, Y. Cui, N. Zhou, J. Shen, Near-infrared light-mediated photodynamic/photothermal therapy nanoplatform by the assembly of $\mathrm{Fe}_{3} \mathrm{O}_{4}$ carbon dots with graphitic black phosphorus quantum dots, Int. J. Nanomed. 13 (2018) 2803-2819.

[42] Z. Qin, S. Du, Y. Luo, Z. Liao, F. Zuo, J. Luo, D. Liu, Hydrothermal synthesis of superparamagnetic and red luminescent bifunctional $\mathrm{Fe}_{3} \mathrm{O}_{4} @ \mathrm{Mn}^{2+}$-doped NaYF $4: \mathrm{Yb} / \mathrm{Er}$ core@shell monodisperse nanoparticles and their subsequent ligand exchange in water, Appl. Surf. Sci. 378 (2016) 174180.

[43] W. Chen, J. Ouyang, H. Liu, M. Chen, K. Zeng, J. Sheng, L. Deng, Black phosphorus nanosheetbased drug delivery system for synergistic photodynamic/photothermal/chemotherapy of cancer, Adv. Mater. 29 (2017) 1603864.

[44] B. Liu, C. Li, P. Yang, Z. Hou, J. Lin, 808-nm-light-excited lanthanide-doped nanoparticles: rational design, luminescence control and theranostic applications, Adv. Mater. 29 (2017) 1605434.

J. Shen, L.D. Sun, Y.W. Zhang, C.H. Yan, Superparamagnetic and upconversion emitting 
$\mathrm{Fe}_{3} \mathrm{O}_{4} / \mathrm{NaYF}_{4}: \mathrm{Yb}$,Er hetero-nanoparticles via a crosslinker anchoring strategy, Chem. Commun. 46 (2010) 5731-5733.

[46] F. Wu, M. Zhang, X. Chu, Q. Zhang, Y. Su, X. Yi, Black phosphorus nanosheets-based nanocarriers for enhancing chemotherapy drug sensitiveness via depleting mutant p53 and resistant cancer multimodal therapy, Chem. Eur. J. 370 (2019) 387-399.

[47] W. Tang, W. Fan, W. Zhang, Wet/Sono-chemical synthesis of enzymatic two-dimensional $\mathrm{MnO}_{2}$ nanosheets for synergistic catalysis-enhanced phototheranostics, Adv. Mater. (2019) 1900401.

[48] Z.L. Dong, H. Gong, M. Gao, W. Zhu, X.Q. Sun, L.Z. Feng, T. Fu, Y.G. Li, Z. Liu, Polydopamine nanoparticles as a versatile molecular loading platform to enable imaging-guided cancer combination therapy, Theranostics 6 (2016) 1031-1042.

[49] Q.Y. Jia, J.C. Ge, W.M. Liu, X.L. Zheng, S.Q. Chen, Y.M. Wen, H.Y. Zhang, P.F. Wang, A magnetofluorescent carbon dot assembly as an acidic $\mathrm{H}_{2} \mathrm{O}_{2}$-driven oxygenerator to regulate tumor hypoxia for simultaneous bimodal imaging and enhanced photodynamic therapy, Adv. Mater. 30 (2018) 1706090.

[50] H.T. Bi, Y.L. Dai, P. Yang, J.T. Xu, D. Yang, S.L. Gai, F. He, G.H. An, C.N. Zhong, J. Lin, Glutathione and $\mathrm{H}_{2} \mathrm{O}_{2}$ consumption promoted photodynamic and chemotherapy based on biodegradable $\mathrm{MnO}_{2}$ Pt@Au25 nanosheets, Chem. Eng. J. 356 (2019) 543-553.

[51] W.S. Chen, J. Ouyang, H. Liu, M. Chen, K. Zeng, J.P. Sheng, Z.J. Liu, Y.J. Han, L.Q. Wang, J. Li, L. Deng, Y.N. Liu, S.J. Guo, Black phosphorus nanosheet-based drug delivery system for synergistic photodynamic/photothermal/chemotherapy of cancer, Adv. Mater. 29 (2017) 1603864.

[52] L Ma, S Feng, C Fuente-Núñez, R. E. Hancock, X.N. Lu, Development of molecularly imprinted polymers to block quorum sensing and inhibit bacterial biofilm formation, ACS Appl. Mater. Interfaces 10.22 (2018) 18450-18457.

[53] H. Huang, L.Z. He, W.H. Zhou, G.B. Qu, J.H. Wang, N. Yang, J. Gao, T.F. Chen, P.K. Chu, X.F. $\mathrm{Yu}$, Stable black phosphorus $/ \mathrm{Bi}_{2} \mathrm{O}_{3}$ heterostructures for synergistic cancer radiotherapy, Biomaterials 171 (2018) 12-22.

[54] H. Shi, R.Q. Yan, L.Y. Wu, Y.D. Sun, S. Liu, Z.Y. Zhou, J. He, D.J. Ye, Tumor-targeting CuS nanoparticles for multimodal imaging and guided photothermal therapy of lymph node metastasis, Acta Biomater. 72 (2018) 256-265.

[55] Y. Shen, L. Liang, J. Zhang, Z. Li, J. Yue, J. Wang, X. Xu, Interference-free surface-enhanced Raman scattering nanosensor for imaging and dynamic monitoring of reactive oxygen species in mitochondria during photothermal therapy, Sens. Actuators, B 285 (2019) 84-91.

[56] D. Yang, G.X. Yang, P. Yang, R.C. Lv, S. Gai, C.X. Li, F. He, J. Lin, Assembly of Au plasmonic photothermal agent and iron oxide nanoparticles on ultrathin black phosphorus for targeted photothermal and photodynamic cancer therapy, Adv. Funct. Mater. 27 (2017) 1700371.

[57] J. Xu, W. Han, T. Jia, S. Dong, H. Bi, D. Yang, F. He, P. Yang, Bioresponsive upconversion nanostructure for combinatorial bioimaging and chemo-photothermal synergistic therapy, Chem. Eur. J. 342 (2018) 446-457. 